\title{
Thin chip microsprayer system coupled to quadrupole time-of-flight mass spectrometer for glycoconjugate analysis
}

\author{
Alina D. Zamfir, $\uparrow^{* a}$ Niels Lion, $\dagger^{b}$ Željka Vukelić, ${ }^{c}$ Laura Bindila, ${ }^{a}$ Joël Rossier, ${ }^{d}$ Hubert H. Girault ${ }^{b}$ and \\ Jasna Peter-Katalinić*a
}

Received 1st September 2004, Accepted 9th November 2004

First published as an Advance Article on the web 22nd December 2004

DOI: $10.1039 / \mathrm{b} 413282 \mathrm{c}$

\begin{abstract}
A thin chip polymer-based microsprayer has been coupled to a hybrid quadrupole time-of-flight mass spectrometer (QTOF MS) and introduced in carbohydrate research. The feasibility of the approach is demonstrated for mapping, sequencing and structural elucidation of glycoconjugates originating from human body fluids and tissues such as a glycopeptide mixture from normal human urine and an isolated and purified GT1 ganglioside fraction from normal adult human brain. The optimization procedure required by each glycoconjugate category is described and the advantages of the system in terms of flexibility and adaptability to QTOF MS, stability of the ESI MS signal, carbohydrate ionization and sequencing, sensitivity, speed of analysis and sample consumption are discussed.
\end{abstract}

\section{Introduction}

In the post-genome era, mass spectrometry (MS) has developed continuously as one of the most powerful analytical techniques for structural elucidation of molecules originating from biological matrices. The potential of MS for highlysensitive structural bioanalyses increased significantly after the introduction of chip-based electrospray ionization (ESI) and matrix assisted laser desorption/ionization (MALDI) methods and the possibility of sequencing complex ionic species by highly efficient dissociation techniques based on multiple stage MS $\left(\mathrm{MS}^{n}\right)$. In particular, in proteomics and glycoproteomics, nanoESI $\mathrm{MS}^{n}$ in the positive as well as in the negative ion mode was shown to be capable of sequencing minute amounts of biological material thus providing straightforward information on various structure determinants. ${ }^{1-5}$

Miniaturized analytical instrumentation is attracting growing interest in chemical, biochemical and structural analysis. Nowadays, various mass spectrometer configurations are interfaced to the integrated micro- and nanosystems or to devices providing either robotized sample infusion or a combination of automated sample delivery and chip-based ionization. The potential of these modern systems is considerably broadening the area of MS applicability in life sciences. The option of miniaturized devices for sample infusion into MS is driven by several technical, analytical and economical advantages such as: (1) simplification of the laborious chemical and biochemical strategies required currently for MS research; (2) high throughput nanoanalysis/identification of biomolecules; (3) elimination of the time-consuming optimization procedures; (4) an increase in the sensitivity by drastic reduction of the sample and reagent consumption, sample handling and potential sample loss; (5) high reproducibility

$\uparrow$ Equal contribution.

*zamfir@uni-muenster.de (Alina D. Zamfir)

jkp@uni-muenster.de (Jasna Peter-Katalinić) of the experiments; (6) the potential to discover novel biologically-relevant structures due to increased ionization efficiency; (7) high signal-to-noise ratio; (8) reduced in-source fragmentation leading to a significant increase of the precision in the identification of unknown analytes in biological matrices; (9) flexibility and a broad area of applicability; (10) low cost of analysis and chip production; (11) the possibility for unattended high-throughput experiments reducing man power and intervention; (12) elimination of possible crosscontamination and carry-overs; (13) flexibility for different configurations and analyses, upgrading and modifications; (14) minimal infrastructure requirements for optimal functioning; (15) reduction of the ion source size facilitating manipulation and efficient ion transfer by precise positioning towards the MS sampling orifice.

Most of the commercially available chip-based devices for MS sample delivery are fabricated in glass or silicon using a variety of semiconductor methods including photolithography and deep ion reactive etching. In combination with automated sample manipulation and infusion, such systems found broad applicability in protein/peptide analysis, ${ }^{6,7}$ drug development, ${ }^{8,9}$ metabolite ${ }^{10}$ and carbohydrate screening. ${ }^{11,12}$

In recent years, the progress in polymer-based microsprayer systems was promoted by development of simpler methods for accurate plastic replications and ease to create lower-cost disposable chips.

Several approaches for interfacing MS to polymer chips providing flow rates which include them in categories from nano- to microsprayers were reported. ${ }^{13-15}$ Different MS configurations $^{16-19}$ such as single and triple quadrupole MS ion trap and ultra high resolution Fourier-transform ion cyclotron resonance mass spectrometry (FTICR MS) were adapted to polymer-based chip ESI interfacing and optimized mostly for proteomic surveys.

Here we report the introduction of a robust thin chip polymer-based microsprayer system in combination with quadrupole time-of-flight tandem mass spectrometry (QTOF 
MS-MS) in carbohydrate research. For the first time to our knowledge the feasibility of such an approach for mapping, sequencing and structural elucidation of this biopolymer category is demonstrated.

The novel mass spectrometric strategy was applied to glycoconjugates originating from human body fluids and tissues such as a glycopeptide mixture from normal human urine and an isolated and purified GT1 ganglioside fraction extracted from normal adult human brain. The optimization procedure required for each type of glycoconjugate is described. Moreover, the advantages of the system in terms of flexibility and adaptability to QTOF MS, stability of the ESI MS signal, carbohydrate ionization and sequencing, sensitivity, speed of analysis and sample consumption are here discussed.

\section{Experimental}

\section{Biological glycoconjugate samples}

Mixture of $\boldsymbol{O}$-glycosylated amino-acids and peptides from normal human urine. The complex mixture of $O$-glycosylated amino-acids and peptides investigated in this work was obtained and partially purified previously from urine of a healthy subject. The extraction, separation and purification procedure was described by us previously. ${ }^{20,21}$ The sample is denoted Ty. Stock solutions of the Ty mixture at $1 \mathrm{mg} \mathrm{ml}^{-1}$ were prepared by dissolving the dried material in $100 \% \mathrm{MeOH}$ and freezing it at $-20{ }^{\circ} \mathrm{C}$. Dilution of the stock solutions in pure $\mathrm{MeOH}$ yielded working aliquots at a concentration of 5.0

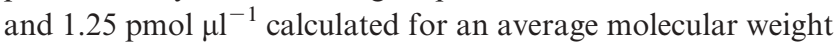
of $2000 \mathrm{Da}$.

GT1 ganglioside fraction from normal adult human cerebrum. Normal adult (45 years of age) human cerebrum, without pathological signs according to morphoanatomical and histopathological examination was obtained from the Department of Forensic Medicine, Faculty of Medicine, University of Zagreb, Croatia. Permission for experiments with human tissue for scientific purposes was obtained from The Ethical Commission of the University of Zagreb, Croatia, under Project no. 108120 by the Ministry of Science and Technology of the Republic of Croatia.

The brain tissue was weighed and stored at $-20{ }^{\circ} \mathrm{C}$ until the extraction procedure. The native fraction of GT1 gangliosides was extracted, isolated and purified in our laboratory as described in detail elsewhere. ${ }^{22}$ The ganglioside sample, denoted GT1, was dried in a Speed Vac SPD 111V system (Savant, Düsseldorf, Germany). For microchip ESI MS analysis, a stock solution of the sample at approximately $1 \mathrm{mg} \mathrm{ml}^{-1}$ was prepared by dissolving the dried material in methanol to be stored at $-20{ }^{\circ} \mathrm{C}$. Dilution of the stock solution in $100 \%$ methanol yielded the working aliquots at a concentration of approximately $5 \mathrm{pmol} \mu \mathrm{l}^{-1}$ of ganglioside extract.

\section{Reagents and materials}

Methanol was obtained from Merck (Darmstadt, Germany) and used without further purification. Distilled and deionized water (Milli-Q water systems Millipore, Bedford, MA, USA) was used for the preparation of the sample solutions. Sample solutions were dried in a SpeedVac SPD 111V system from Savant (Düsseldorf, Germany) and centrifuged in an Eppendorf $5415 \mathrm{C}$ centrifuge (Hamburg, Germany).

\section{Instrumentation}

Mass spectrometry. Mass spectrometry was performed on an orthogonal hybrid quadrupole time-of-flight mass spectrometer (QTOF ${ }^{(i m}$ Micromass, Manchester, U.K.) in Micromass Z-spray geometry. The QTOF mass spectrometer is interfaced to a PC computer running the MassLynx software under the Windows N.T. system to control the instrument, acquire and process MS data. Nitrogen was used as dissolvation gas and the source block temperature was kept at $80{ }^{\circ} \mathrm{C}$. The sampling cone potential and voltage applied on the microsprayer were adjusted to provide an optimal ionization, minimal in-source fragmentation and a high and sustained electrospray signal.

Tandem mass spectrometry was performed by collisioninduced dissociation (CID) at low energies using $\mathrm{Ar}$ as a collision gas. In order to obtain a maximum coverage of sequence ions, the collision energy was kept at $40 \mathrm{eV}$ for glycopeptide sequencing and adjusted during the ongoing experiment from 40 to $70 \mathrm{eV}$ for sequencing of GT1 species.

All mass spectra were acquired in the negative ion mode. For mapping and sequencing of glycoconjugates, the negative ion detection was shown to be advantageous in comparison with the positive ion mode. ${ }^{23,24}$ In particular, the assignment of already known or previously unknown components in biological mixtures is easier due to a lower degree of heterogeneity caused by cation attachment to molecular and/or fragment ions. $^{23-26}$

After acquisition, the spectra were calibrated using sodium iodide as a calibrant.

Thin chip microsprayer system. The microchip fabrication has been described previously. ${ }^{27}$ Basically, a photoresist is patterned on a $75 \mu \mathrm{m}$ thick, copper-coated polyimide foil through a printed slide acting as a mask. The photoresist is then developed, and chemical etching is then used to remove the deprotected copper where microchannels are to be patterned. Polyimide is plasma-etched to the desired depth. The final microchannels are $120 \mu \mathrm{m}$ wide, $45 \mu \mathrm{m}$ deep (nearly "half moon" cross section), with gold-coated microelectrodes placed at the bottom of the microchannel. A $35 \mu \mathrm{m}$ polyethylene-polyethylene terephthalate is laminated to close the channels. Finally, one end of each channel is manually cut in a tip shape, so that the outlet of the microchannel is located on the edge of the chip, as described by Gobry et al. ${ }^{28}$ For sample dispensing, a reservoir is pasted over the inlet of the microchannel, or the chip is sandwiched in a home-made chip holder with an integrated reservoir.

The whole chip/reservoir assembly was mounted to the QTOF MS. In order to realize the electrical contact to the ESI power supply, the ESI QTOF source was removed and the chip system was directly connected to the ESI high voltage plate, which is a fixed part of the QTOF conventional Micromass ESI source. Exchange between the original source and chip 
system interface did not require any definitive dismantling or special mechanical modifications to the original assembly and no further modifications of the QTOF instrument were necessary. The position of the chip emitter was adjusted in the vicinity of the entrance hole of the sampling cone by the source assembly, which can be manipulated in the $x, y$ and $z$ direction via micrometer screws. The microsprayer tip was placed at a distance less than $5 \mathrm{~mm}$, although fine positioning of the microsprayer tip turned out not to be a critical parameter.

The electrical contact was ensured by a conductive wire with one terminal connected to the chip electrode and the other fixed on the ESI high voltage plate. The spray could be initiated at values of $2-3 \mathrm{kV}$, in the negative ion mode, applied to the nanoESI plate and $80-100 \mathrm{~V}$ applied to the sampling cone without the need for nebulizer gas. The chip microchannel was flushed with methanol between two consecutive experiments with the same sample. For all experiments, desolvation at $80{ }^{\circ} \mathrm{C}$ under drying gas was effective, so that the chip unit could be left connected to the mass spectrometer during the channel rinse.

For each sample a fresh chip was used thus any contamination was prevented. Under the same ESI QTOF MS conditions, the in-run reproducibility of the experimental data in terms of sensitivity, spray stability, number of detected components/fragments, ion intensity and charge state was almost $100 \%$ while the day-to-day reproducibility was 95$100 \%$ with a maximum mass deviation of $10 \mathrm{ppm}$, which is within in the normal range for a QTOF I instrument. The minimum number of replicates for an experiment was 3 .

The flow rate generated under the employed source and

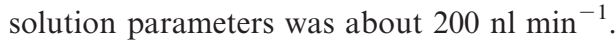

\section{Results and discussion}

\section{Determination of $\boldsymbol{O}$-GalNAc-Ser/Thr expression in normal human urine}

In human urine, carbohydrates are catabolic products excreted as either oligosaccharides or glycopeptides and their concentration, amount and structure is known to vary under different physiological and/or pathological conditions. For this reason, screening and identification of glycopeptide expression in human urine is of major biological importance.

A Ty mixture of $O$-GalNAc glycosylated amino acids and peptides extracted from normal human urine was dissolved in pure methanol to a concentration of $5 \mathrm{pmol} \mu \mathrm{l}^{-1}$. An aliquot of $10 \mu \mathrm{l}$ was dispensed into a reservoir pasted over the inlet of the chip microchannel. The $(-)$ ion mode electrospray process was initiated at $2.8 \mathrm{kV}$ applied to the ESI high voltage plate and $100 \mathrm{~V}$ potential of the sampling cone. Under these welldefined conditions a constant and stable spray accompanied by a high intensity of the total ion current was generated. In Fig. 1a, the (-)chipESI QTOF MS1 of the Ty sample at a 5 pmol $\mu^{-1}$ concentration is presented. The signal was acquired over 20 scans (40 seconds) which at the flow rate of about $200 \mathrm{nl} \mathrm{min}-1$ is equivalent to a sample consumption of 0.66 pmol. The spectrum combined over 20 scans exhibits a high signal/noise ratio and 26 different saccharide components. 15 species detected as singly and/or doubly charged ions are expressing the $O$-GalNAc-Ser/Thr core-motif extended by either sialylation or fucosylation. The mixture is dominated by Ser- and Thr- linked disialo saccharides with chain lengths ranging from tetra- to octasaccharide. The monosialylated glycopeptides are also well represented in the spectrum in Fig. 1a, being detected as singly charged ions at $\mathrm{m} / \mathrm{z} 760.12$, NeuAcHexHexNAc-Ser, and 774.12, NeuAcHexHexNAcThr. Mono- and disialylated free oligosaccharides are observed as singly charged ions at $\mathrm{m} / \mathrm{z} 470.03$ corresponding to disaccharide NeuAcHex, $m / z \quad 673.12$ assigned to NeuAcHexHexNAc trisaccharide and $\mathrm{m} / \mathrm{z} 964.16$ assigned to

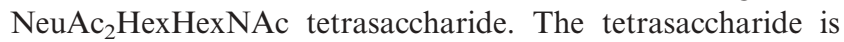
also observed as a doubly charged ion at $\mathrm{m} / \mathrm{z} 481.09$.

Interestingly, two new structures, not detectable before by any MS-related methods, are visible as doubly charged ions at $\mathrm{m} / \mathrm{z} 1069.19$ and 1076.20. The species could originate from glycopeptides with extended and further modified chains.

In order to test the limit of microchip sensitivity for glycoconjugate detection, Ty solution was diluted in pure methanol yielding an aliquot at $1.25 \mathrm{pmol} \mu \mathrm{l}^{-1}$ concentration. In Fig. 1b, the (-) microchip ESI QTOF MS1 of the Ty

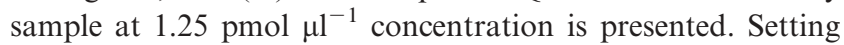
the same source parameters, the signal was acquired over 20 scans, which is equivalent to $0.16 \mathrm{pmol}$ sample consumption for this experiment. Even under these restrictive concentration conditions, a fair signal/noise ratio and 13 different components in the mixture could be identified.

In Table 1 the assignment of the major species present in the Ty sample and detected by microchip ESI QTOF MS1 is given.

An interesting feature of the spectra in Fig. 1 is that at $100 \mathrm{~V}$ cone potential, the charge distribution is not shifted towards lower values as observed in the capillary-based ESI QTOF MS experiments, the intensity of the signals corresponding to doubly charged ions being the highest. Moreover, the advantage of the microchip ESI MS regarding the minimization of the in-source decay is clearly illustrated in Fig. 1 where the in-source fragmentation of molecular ions is not observed at such a high value of the sampling cone potential.

To investigate the possibility of performing rapid and accurate glycopeptide sequencing by MS/MS using the polymer chip for sample infusion, the doubly charged ion at $\mathrm{m} / \mathrm{z} 532.08$, assigned according to the $\mathrm{m} / \mathrm{z}$ value to the already known structure of $\mathrm{NeuAc}_{2} \mathrm{HexHexNAc-Thr,} \mathrm{was} \mathrm{isolated}$ and submitted to low-energy CID (-) microchip ESI QTOF MS/MS. Collision energy, collision gas pressure and precursor ion isolation parameters were carefully adjusted to provide the full set of structural information upon the molecule. The product ion spectrum of the doubly charged ion at $\mathrm{m} / \mathrm{z} 532.08$ is depicted in Fig. 2. The spectrum was obtained after 30 scans (1 min) of signal acquisition, resulting in a sample consumption of 1.23 pmols.

The product ion spectrum exhibits a high signal/noise ratio and reveals a significant number of fragment ion fingerprints for detailed structural elucidation of the molecule. The abundant fragment ion detected as a singly charged ion at $\mathrm{m} / \mathrm{z}$ 483.07, assigned to Hex-HexNAc-Thr, corresponds to the loss of both sialic acid moieties from the precursor ion. The stripping of only one sialic acid moiety is indicated by the doubly charged ion at $\mathrm{m} / z 386.56$ and the singly charged at $\mathrm{m} / \mathrm{z}$ 

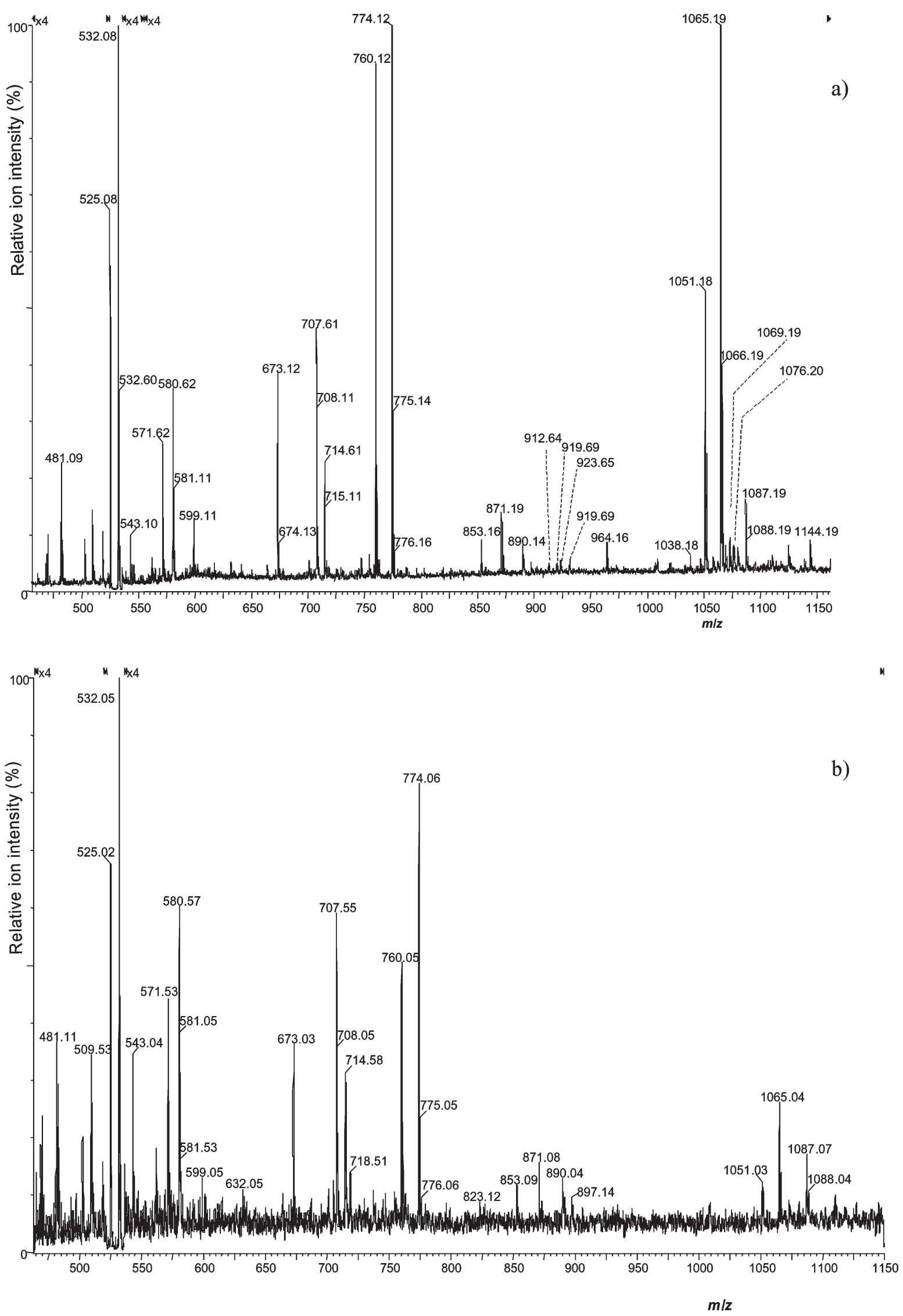

Fig. 1 Microchip ESI QTOF MS1 of the Ty mixture of $O$-glycosylated amino acids and peptides from human urine. ESI voltage $2.8 \mathrm{kV}$; sampling cone potential $100 \mathrm{~V}$; signal acquisition 20 scans. Solvent: $\mathrm{MeOH}$; average sample concentration: (a) 5 pmol $\mu \mathrm{l}^{-1}$; (b) $1.25 \mathrm{pmol}^{-1} \mathrm{l}^{-1}$. 
Table 1 The compositional mapping of the purified native Ty mixture of glycopeptides from normal human urine as detected by (-) microchip ESI QTOF MS1

\begin{tabular}{|c|c|c|c|c|}
\hline$m / z$ (monoisotopic) & Charge state & Putative structure & $5 \mathrm{pmol} \mathrm{l}^{-1}$ & $1.25 \mathrm{pmol}_{\mu} \mathrm{l}^{-1}$ \\
\hline 470.05 & $1-$ & NeuAcHex & + & + \\
\hline 481.11 & $2-$ & $\mathrm{NeuAc}_{2} \mathrm{HexHexNAc}$ & + & + \\
\hline 525.08 & $2-$ & $\mathrm{NeuAc}_{2} \mathrm{HexHexNAc-Ser}$ & + & + \\
\hline 532.08 & $2-$ & $\mathrm{NeuAc}_{2} \mathrm{HexHexNAc-Thr}$ & + & + \\
\hline 543.10 & $2-$ & 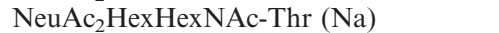 & + & + \\
\hline 571.62 & $2-$ & $\mathrm{NeuAc}_{2} \mathrm{HexHexNAc-Thr-Pro-} \mathrm{H}_{2} \mathrm{O}$ & + & + \\
\hline 580.62 & $2-$ & $\mathrm{NeuAc}_{2} \mathrm{HexHexNAc-Thr-Pro}$ & + & + \\
\hline 673.12 & $1-$ & NeuAcHexHexNAc & + & + \\
\hline 707.61 & $2-$ & $\mathrm{NeuAc}_{2} \mathrm{Hex}_{2} \mathrm{HexNAc}_{2}$-Ser & + & + \\
\hline 714.61 & $2-$ & $\mathrm{NeuAc}_{2} \mathrm{Hex}_{2} \mathrm{HexNAc}_{2}-\mathrm{Thr}$ & + & + \\
\hline 760.12 & $1-$ & NeuAcHexHexNAc-Ser & + & + \\
\hline 774.12 & $1-$ & NeuAcHexHexNAc-Thr & + & + \\
\hline 853.16 & $1-$ & NeuAcHexHexNAc-Thr-Pro $-\mathrm{H}_{2} \mathrm{O}$ & + & + \\
\hline 871.19 & $1-$ & NeuAcHexHexNAc-Thr-Pro & + & + \\
\hline 890.14 & $2-$ & $\mathrm{NeuAc}_{2} \mathrm{Hex}_{3} \mathrm{HexNAc}_{3}$-Ser & + & + \\
\hline 897.17 & $2-$ & $\mathrm{NeuAc}_{2} \mathrm{Hex}_{3} \mathrm{HexNAc}_{3}-\mathrm{Thr}$ & + & + \\
\hline 912.64 & $2-$ & $\mathrm{NeuAc}_{2} \mathrm{Hex}_{2} \mathrm{HexNAc}_{4}-\mathrm{Ser}-\mathrm{H}_{2} \mathrm{O}(\mathrm{Na})$ & + & \\
\hline 919.69 & $2-$ & $\mathrm{NeuAc}_{2} \mathrm{Hex}_{2} \mathrm{HexNAc}_{4}-\mathrm{Thr}-\mathrm{H}_{2} \mathrm{O}(\mathrm{Na})$ & + & \\
\hline 923.65 & $2-$ & $\mathrm{NeuAc}_{2} \mathrm{Hex}_{2} \mathrm{HexNAc}_{4}-\mathrm{Ser}-\mathrm{H}_{2} \mathrm{O}(2 \mathrm{Na})$ & + & \\
\hline 930.67 & $2-$ & $\mathrm{NeuAc}_{2} \mathrm{Hex}_{2} \mathrm{HexNAc}_{4}-\mathrm{Thr}-\mathrm{H}_{2} \mathrm{O}(2 \mathrm{Na})$ & + & \\
\hline 964.16 & $1-$ & $\mathrm{NeuAc}_{2} \mathrm{HexHexNAc}$ & + & \\
\hline 1051.18 & $1-$ & $\mathrm{NeuAc}_{2} \mathrm{HexHexNAc-Ser}$ & + & + \\
\hline 1065.19 & $1-$ & $\mathrm{NeuAc}_{2} \mathrm{HexHexNAc-Thr}$ & + & + \\
\hline 1069.19 & $2-$ & n.a. & + & \\
\hline 1076.20 & $2-$ & n.a. & + & \\
\hline 1087.19 & $2-$ & n.a. & + & + \\
\hline 1144.19 & $1-$ & $\mathrm{NeuAc}_{2} \mathrm{HexHexNAc-Thr-Pro}-\mathrm{H}_{2} \mathrm{O}$ & + & \\
\hline
\end{tabular}

774.13 along with the ions obtained by further neutral loss of $\mathrm{H}_{2} \mathrm{O}$ at $\mathrm{m} / z 756.10$ and of $\mathrm{CO}_{2}$ at $\mathrm{m} / z$ 730.13. The trisaccharide ion $\mathrm{C}_{3} / \mathrm{B}_{1 \beta}$ at $m / z 673.06$ is assigned to NeuAc-Hex-HexNAc. Of particular interest for the localization of the sialic acid residue are the singly charged ions at $\mathrm{m} / \mathrm{z} 470.05$, assigned to NeuAc-Hex $\left(\mathrm{C}_{2 \alpha}\right)$ and at $m / z$ 493.07, corresponding to NeuAcHexNAc $\left(\mathrm{C}_{3} / \mathrm{C}_{2 \alpha}\right)$. These ions indicating the attachment of the NeuAc moiety represent valuable diagnostic elements for the sialylation pattern of the core $1 O$-GalNAc mucine molecules. In addition, the ion generated only by the glycosidic-amino acid bond cleavage, with retention of sialic acid entities, is evidenced by the doubly charged ion at $\mathrm{m} / \mathrm{z} 481.60$, assigned to $(\mathrm{NeuAc})$ Hex-HexNAc $\left(\mathrm{C}_{3}\right)$. In the spectrum in Fig. 2, two relevant ring cleavage ions at $\mathrm{m} / \mathrm{z} 350.04$ and at $\mathrm{m} / \mathrm{z} 306.08$ corresponding to ${ }^{0,4} \mathrm{~A}_{2}$ and ${ }^{0,4} \mathrm{~A}_{2}-\mathrm{CO}_{2}$ are detected. According to our previous studies ${ }^{29,30}$ these cleavages are diagnostic for the NeuAc $\alpha 2-6$ linkage.

\section{Characterization of GT1 ganglioside fraction from normal adult human cerebrum}

Gangliosides are sialylated glycosphingolipids (GSLs) consisting of mono- to poly- sialylated oligosaccharide chains of variable length attached to the ceramide portion. For analysis of complex ganglioside mixtures from biological sources by MS methods, either with ESI or MALDI, specific conditions related to the ionization process and a reliable assessment of the heterogeneity of composition, followed by identification of the molecule architecture, are required.

Optimization of a procedure for capillary-based (-)nanoESI QTOF MS and tandem MS for characterization of native ganglioside mixture from brain tissues was recently reported by us. ${ }^{22,33,34}$ In these studies, requirements for elevated values of ionization parameters and an extended time for signal acquisition implying relatively high sample consumption for identification and structural elucidation were defined.

To test the feasibility and advantages of the microchip ESI QTOF MS analysis concerning information that could be provided by both MS1 and MS/MS, as well as to define corresponding appropriate conditions for the GSL molecular class detection and structural characterization, a rather structurally complex polysialylated ganglioside fraction, GT1, was chosen as the testing sample. The analyzed GT1 ganglioside fraction, showing migration properties of GT1b species in high performance thin-layer chromatography (HPTLC), was isolated from the total native ganglioside mixture purified from adult human cerebrum.

For (-) microchip ESI QTOF MS analysis, GT1 sample was dissolved in pure $\mathrm{MeOH}$ to a concentration of $5 \mathrm{pmol} \mu \mathrm{l}^{-1}$ and an aliquot of $10 \mu \mathrm{l}$ was loaded into the microchip reservoir. To optimize the ionic current value, the $(-)$ ESI voltage and cone potential were increased from zero following a ramping procedure. The maximum current value was obtained at $3 \mathrm{kV}$ ESI and $100 \mathrm{~V}$ applied on the sampling cone. In Fig. 3a the total ion current (TIC) of GT1 acquired for $6 \mathrm{~min}$ is presented. The TIC profile indicates a sustained spray varying only by ESI source parameter variation, as well as an efficient ionization at $3 \mathrm{kV}$ ESI and $100 \mathrm{~V}$ sampling cone voltages, proved by the high intensity of ionic current. Starting with $1.5 \mathrm{~min}$, the signal was acquired for 4 minutes under $3 \mathrm{kV}$ and $100 \mathrm{~V}$, to give a constant ESI signal documented by the flat region of the TIC. By combining in progress over all TIC 


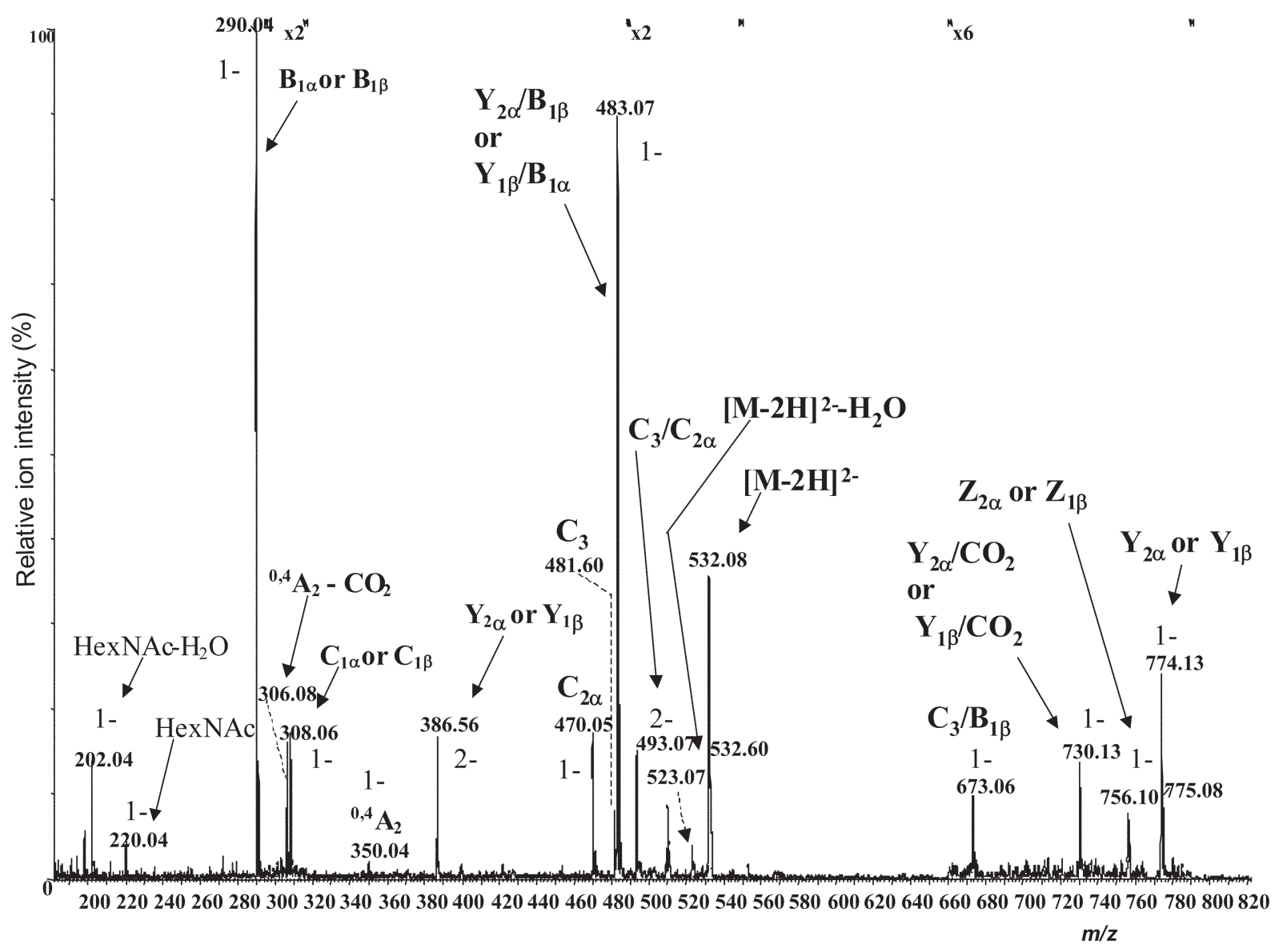

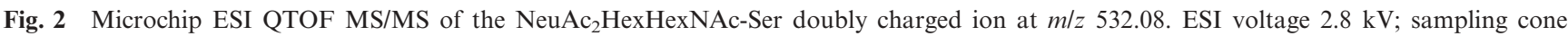

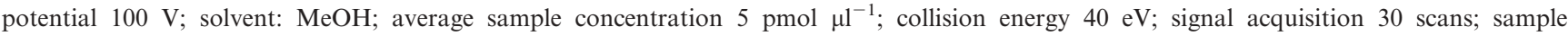
consumption 1.23 pmols. The nomenclature for assignment of fragment ions is according to Domon and Costello nomenclature. ${ }^{31,32}$

scans of constant current intensity, a spectrum of high signal/ noise was obtained (Fig. 4) however, a fair signal/noise ratio was already visible after 60 scans ( 30 seconds) of acquisition.

A reproducible compositional mapping of molecular components in the GT1 fraction mixture was obtained from both sets of formed molecular ions, the triply and the doubly charged related to gangliosides containing a number of lipid variants (Table 2). The enlarged area of the spectrum $(\mathrm{m} / \mathrm{z}$ range 1030-1125), containing a rich pattern of doubly charged molecular ions all attributed to GT1 species, is presented in Fig. 4b. In particular, each specific GT1 species is characterized exclusively by one triply charged $\left[\mathrm{M}-3 \mathrm{H}^{+}\right]^{3-}$ ion, while the corresponding doubly charged $\left[\mathrm{M}-2 \mathrm{H}^{+}\right]^{2-}$ ion is mostly accompanied by the same charge state $\left[\mathrm{M}+\mathrm{Na}-3 \mathrm{H}^{+}\right]^{2-}$ and/or $\left[\mathrm{M}-2 \mathrm{H}^{+}\right]^{2-}-\mathrm{H}_{2} \mathrm{O}$ ions.

The presence of three minor GT1 molecular species is documented only by very low intensity ion signals at $\mathrm{m} / \mathrm{z}$ 1094.20, 1108.20 and 1109.21, which can be assigned by calculation to GT1 (d18:1/21:1), GT1 (d18:1/23:1) and GT1 (d18:1/23:0), respectively.

As is obvious from the spectrum pattern, only a low level of in-source fragmentation occurs during the MS1 ionization process, according to the ions assigned to disialylated product ions resulting from the cleavage of one sialic acid residue from the intact trisialylated GT1 species. The NeuAc residue linked to the terminal Gal residue as a monosialo group is known to be more susceptible to enzymatic cleavage, such as that by $V$. cholerae neuraminidase. The loss of the NeuAc residue(s) bound to the inner Gal would produce a high extent of disialo ions along with NeuAc-Gg ${ }_{4} \mathrm{Cer}$ (GM1-like) fragment ions, which are not detectable in the spectrum. A significantly lower extent of in-source induced fragmentation using chip-based ESI $v s$. conventional capillary-based ion source has already been observed and documented, ${ }^{35}$ representing one of the major advantages of chip usage in MS analysis.

It is well-known that in the case of ganglioside fragmentation by CID MS/MS, a long signal acquisition time under variable collision energy is necessary to obtain a sufficient abundance of fragment ions for structure elucidation. This approach, though successfully applied by using capillary-based ESI, ${ }^{22,33,34}$ often encounters difficulties associated with spray instability and/or signal interruptions. In the case of microchip ESI MS/MS the triply charged ion detected at $\mathrm{m} / \mathrm{z} 717.50$ could be successfully fragmented. The TIC profile of the sequencing events given in Fig. 3b) documents the stability of the MS/MS signal for each of the employed collision energy values used for this experiment and the corresponding dependence of the ionic current on this parameter. The MS/MS product ion spectrum 

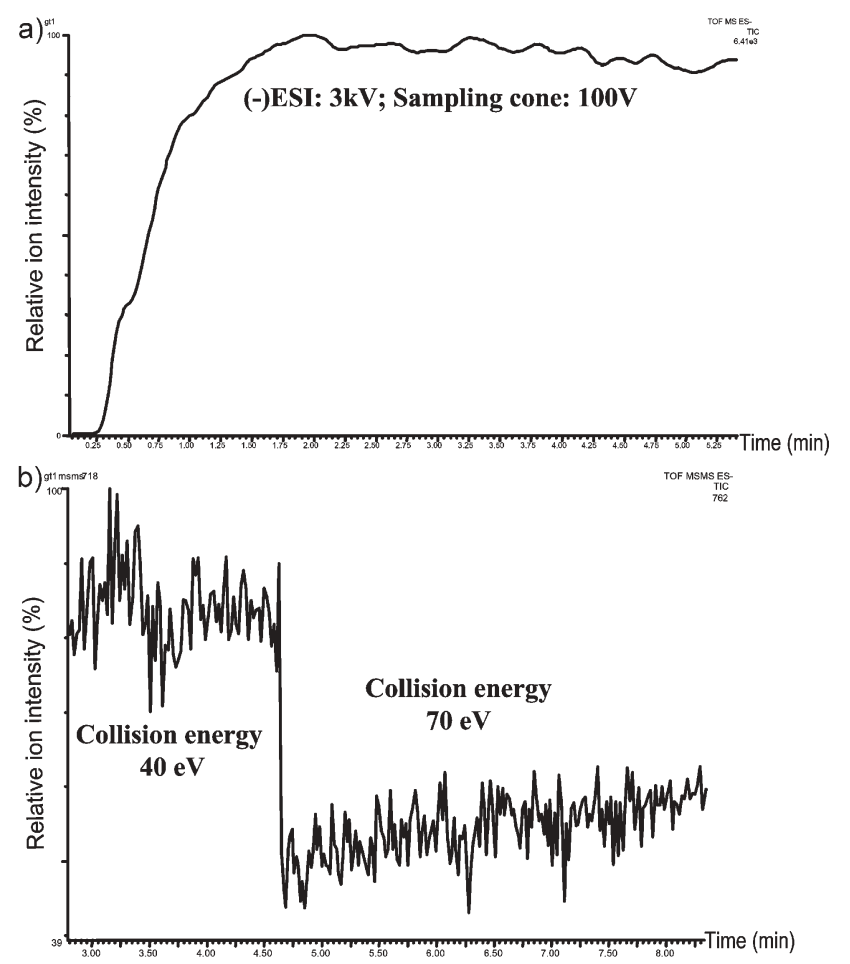

Fig. 3 Microchip ESI QTOF TIC of the GT1 ganglioside fraction from normal adult human brain. Maximal ionic current at $3 \mathrm{kV}$ ESI voltage and $100 \mathrm{~V}$ sampling cone potential. Solvent: $\mathrm{MeOH}$; average sample concentration 5 pmol $\mu \mathrm{l}^{-1}$; (a) MS1 mode; (b) MS/MS mode for the triply charged ion at $\mathrm{m} / \mathrm{z} 717.50$.

of the triply charged precursor ion at $\mathrm{m} / \mathrm{z} 717.63$, corresponding to the GT1 (d18:1/20:0) species is depicted in Fig. 5. The spectrum was acquired under variable energy (VE-CID) ${ }^{36}$ by combining scans at collision energy values of 40 and $70 \mathrm{eV}$. The fragmentation process gave rise to product ions for reliable assignment of the entire carbohydrate sequence of the GT1b isomer species, as well as for characterization of the type of its ceramide portion. However, certain ions indicative for positional isomers, particularly those of GT1 $\alpha$, GT1c and/or even GT1a, in the mixture with GT1b, are present as minor components. The ion assignment in the shown spectrum defines the GT1b product ions as represented in the accompanying fragmentation scheme (Fig. 6).

The triply charged, still highly abundant precursor ion is clearly detectable in the spectrum. As a general characteristic, a predominance of the singly over the doubly and triply charged fragment ions can be pointed out. Also, no sodiated counterparts are observed. The complete sequence of the GT1b is deducible from the ions arising from the non-reducing end of the molecule where dominating $\mathrm{Y}$ type ions are accompanied by $\mathrm{Z}$ and ring cleavage $\mathrm{X}$ type ions appearing at lower abundance, as deduced from the corresponding fragmentation scheme (Fig. 6). The d18:1/18:0 type of ceramide is represented by both $\mathrm{Y}_{0}$ ion and a minor $\mathrm{Z}_{0}$ at $\mathrm{m} / z 592.50$ and 574.55 , respectively. The Glc-Cer sequence is also shown by $\mathrm{Y}_{0}$ and $\mathrm{Z}_{0}$ counterparts at $\mathrm{m} / \mathrm{z} 754.49$ and 736.55 , respectively. The $\mathrm{Y}_{2 \alpha} / \mathrm{B}_{2 \beta}$ ion at $m / z 916.55$ gives rise to the Gal-Glc-Cer sequence which might originate from either
GT1a or GT1b isomer after the respective desialylation of either NeuAc or $\mathrm{NeuAc}_{2}$ residue from the inner Gal residue, or might hypothetically originate from an isomer containing the non-substituted inner Gal (potential GT1d isomer). The ${ }^{2,4} \mathrm{X}_{2 \alpha}$ ion resulting from ring cleavage at the GalNAc residue corresponds to the inner Gal disialylated structure, GalNAc$\left(\mathrm{NeuAc}_{2}\right)$ Gal-Glc-Cer, consistent with the GT1b isomer. The disialylation at the inner Gal residue is finally confirmed by the doubly charged $\mathrm{Y}_{2 \alpha}$ ion at $\mathrm{m} / \mathrm{z} 748.80$, detected at lower abundance. The highly abundant doubly charged signal at $\mathrm{m} / z 931.39$ correspondingly to GT1b isomer, can be attributed to both $Y_{4 \alpha}$ and $Y_{3 \beta}$ ions giving rise to the presence of a disialylated $\mathrm{Gg}_{4}$ Cer sequence; these ions, however, do not indicate the attachment sites of sialic acid residues at the neutral $\mathrm{Gg}_{4}$ core. Except for the $\mathrm{Y}_{2 \alpha}$ and the ${ }^{2,4} \mathrm{X}_{2 \alpha}$ specific for the GT1b isomer, all ions could also arise from other isomers, such as GT1 $\alpha$, GT1a, GT1c or even, the as yet not reliably proven to exist, GT1d isomer. The GT1 corresponding sequence was finally corroborated by the triply charged signal at $\mathrm{m} / \mathrm{z} 643.64$ consistent with 0,2 type ring cleavage at one of the terminal sialic acid residues which, in the case of GT1b species, can be formed from both ${ }^{0,2} \mathrm{X}_{4 \alpha}-2 \mathrm{H}^{+}$and ${ }^{0,2} \mathrm{X}_{3 \beta}-2 \mathrm{H}^{+}$ion.

Further support and deeper insight into the detailed structural composition of the chosen molecular ion is provided by a set of ions formed from the reducing end. In particular, informative $\mathrm{C}$ and $\mathrm{B}$ type ions are present and observed to be similarly favored under the defined conditions. Structurally significant and/or considerably abundant A type ring cleavage ions were not found. The B type ion with the highest intensity at $m / z 290.08$, attributable to both the $\mathbf{B}_{1 \alpha}$ and $\mathbf{B}_{1 \beta}$ linkage cleavages, and the accompanying $\mathrm{C}$ type counterpart ion, specifying both the $C_{1 \alpha}$ and $C_{1 \beta}$ cleavages, characterize the cleaved monosialo residue. The presence of the disialo group is evidenced by the abundant $\mathrm{B}_{2 \beta}$ ion at $m / z 581.13$. The $\mathrm{C}_{2 \alpha}$ ion $(\mathrm{m} / \mathrm{z}$ 470.12) representing the NeuAc-Gal sequence confirms the attachment of the monosialo group to the terminal Gal residue, which is consistent with the GT1b but also the GT1a $\alpha$ structural motif. The signal might potentially include the ions formed from the sialylated inner Gal residue by multiple internal cleavages. The counterpart signals at $\mathrm{m} / \mathrm{z} 673.15$ and 655.21, arising from GT1b as $\mathrm{C}_{3 \alpha}$ and $\mathrm{B}_{3 \alpha}$ ions, respectively, represent both NeuAc-Gal-GalNAc and Gal-GalNAcNeuAc sequences, and could therefore arise from any GT1 isoform. The signal at $\mathrm{m} / \mathrm{z} 364.09$ corresponding to the Gal-GalNAc sequence, assigned to the GT1b internal cleavage $\mathrm{B}_{3 \alpha} / \mathrm{B}_{1 \alpha}$ ion, is however more characteristic for the GT1c isomer (the $\mathrm{B}_{2 \alpha}$ ion) bearing the non-substituted terminal Gal-GalNAc residue. The GT1c isoform is further supported by the structure-specific ion at $\mathrm{m} / \mathrm{z} 966.39$ assigned to the ${ }^{2,4} \mathrm{X}_{2 \alpha}$ ring cleavage ion containing the three sialo structural motif. Finally, the prominent ion at $\mathrm{m} / \mathrm{z} 493.10$ attributed to the B type NeuAc-GalNAc ${ }^{-}$ion can arise exclusively from the GT1 $\alpha$ isoforms (potentially GT1a $\alpha$ and/or GT1b $\alpha$ ), characterized by the sialylation of the GalNAc residue. This ion strongly supports the hypothesized presence of at least one GT1 $\alpha$ isoform in the purified GT1 mixture from human brain, containing the GT1b isomer as the major component. 

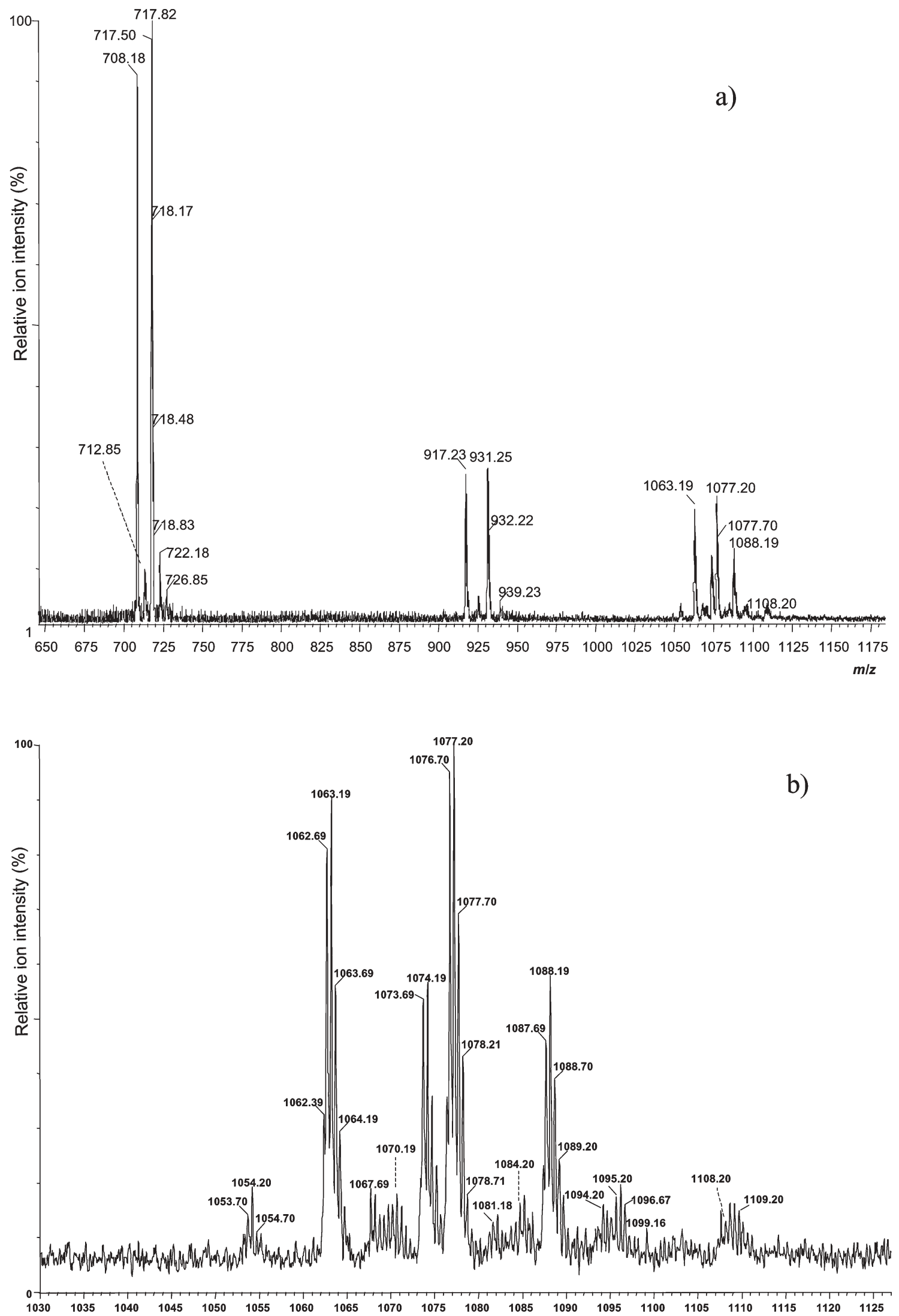

Fig. 4 Microchip ESI QTOF MS1 of the GT1 fraction derived by combining the TIC MS scans at $3 \mathrm{kV}$ ESI voltage and $100 \mathrm{~V}$ sampling cone potential. (a) $m / z$ range: (650-1175); (b) zoom out of the $m / z$ range: (1030-1125). 
Table 2 The compositional mapping of the purified native GT1 ganglioside fraction (exhibiting HPTLC migration properties of the GT1b species) separated from the total ganglioside mixture isolated from adult human brain tissue as detected by (-) microchip ESI QTOF MS1

\begin{tabular}{|c|c|c|}
\hline $\begin{array}{l}\mathrm{m} / \mathrm{z} \\
\text { (monoisotopic) }\end{array}$ & $\begin{array}{l}\text { Type of detected } \\
\text { molecular ion }\end{array}$ & Putative structure \\
\hline 708.18 & {$\left[\mathrm{M}-3 \mathrm{H}^{+}\right]^{3-}$} & GT1 (d18:1/18:0) \\
\hline 1062.69 & {$\left[\mathrm{M}-2 \mathrm{H}^{+}\right]^{2-}$} & \\
\hline 1073.69 & {$\left[\mathrm{M}+\mathrm{Na}^{+}-3 \mathrm{H}^{+}\right]^{2-}$} & \\
\hline 1053.70 & {$\left[\mathrm{M}+2 \mathrm{H}^{+}\right]^{2-}-\mathrm{H}_{2} \mathrm{O}$} & \\
\hline 712.85 & {$\left[\mathrm{M}-3 \mathrm{H}^{+}\right]^{3-}$} & GT1 (d18:1/19:0) \\
\hline 1070.19 & {$\left[\mathrm{M}-2 \mathrm{H}^{+}\right]^{2-}$} & \\
\hline 1081.18 & {$\left[\mathrm{M}+\mathrm{Na}^{+}-3 \mathrm{H}^{+}\right]^{2-}$} & \\
\hline 717.50 & {$[\mathrm{M}-3 \mathrm{H}]^{3-}$} & GT1 (d18:1/20:0) \\
\hline 1076.70 & {$[\mathrm{M}-2 \mathrm{H}]^{2-}$} & \\
\hline 1087.69 & {$[\mathrm{M}+\mathrm{Na}-3 \mathrm{H}]^{2-}$} & \\
\hline 1067.69 & {$\left[\mathrm{M}+2 \mathrm{H}^{+}\right]^{2-}-\mathrm{H}_{2} \mathrm{O}$} & \\
\hline $1094.20^{a}$ & {$\left[\mathrm{M}+\mathrm{Na}^{+}-3 \mathrm{H}^{+}\right]^{2-}$} & GT1 (d18:1/21:1) \\
\hline 722.18 & {$\left[\mathrm{M}-3 \mathrm{H}^{+}\right]^{3-}$} & GT1 (d18:1/21:0) \\
\hline 1084.20 & {$[\mathrm{M}-2 \mathrm{H}]^{2-}$} & \\
\hline 1095.20 & {$\left[\mathrm{M}+\mathrm{Na}^{+}-3 \mathrm{H}^{+}\right]^{2-}$} & \\
\hline 726.85 & {$\left[\mathrm{M}-3 \mathrm{H}^{+}\right]^{3-}$} & GT1 (d18:1/22:0) \\
\hline $1108.20^{a}$ & {$\left[\mathrm{M}+\mathrm{Na}^{+}-3 \mathrm{H}^{+}\right]^{2-}$} & GT1 (d18:1/23:1) \\
\hline $1109.21^{a}$ & {$\left[\mathrm{M}+\mathrm{Na}^{+}-3 \mathrm{H}^{+}\right]^{2-}$} & GT1 (d18:1/23:0) \\
\hline
\end{tabular}

\section{Conclusions}

A thin yet sturdy polymer microchip has been for the first time coupled to QTOF MS and introduced in carbohydrate research. Two complex carbohydrate mixtures containing glycoforms from different biological sources and belonging to different structural systems, a mixture of $O$-glycopeptides from human urine and the isolated GT1 ganglioside fraction from normal adult human brain were explored by this approach. The potential of the methodology developed here is beneficial for glycomics due to particular advantages provided by microchip ESI infusion: (i) a high signal-to-noise ratio within a short scanning time; (ii) a stable spray of biological samples under different solvent and instrumental conditions; (iii) almost 100\% reproducibility of the obtained data under the same experimental conditions; (iv) reduced in-source fragmentation; (v) high sensitivity and resolution in detecting molecular ions; (vi) efficient fragmentation in tandem MS and (vii) the possibility to discover in a single MS experiment new carbohydrate species present in biological mixtures.

Due to the high sensitivity for detection of minor relative to major carbohydrate species in mixtures and other here demonstrated advantages, the microchip ESI QTOF MS has the potential to be introduced in medical diagnostics/pharmacy for comparative compositional analysis of homologous biological glycoconjugate mixtures from either tissues/body fluids or natural products in pharmaceutics. For instance, the microchip ESI QTOF MS analysis of a pathological vs. control glycoconjugate mixture would offer reliable, fast, onestep biochemical diagnosis of diseases. It could be optimized

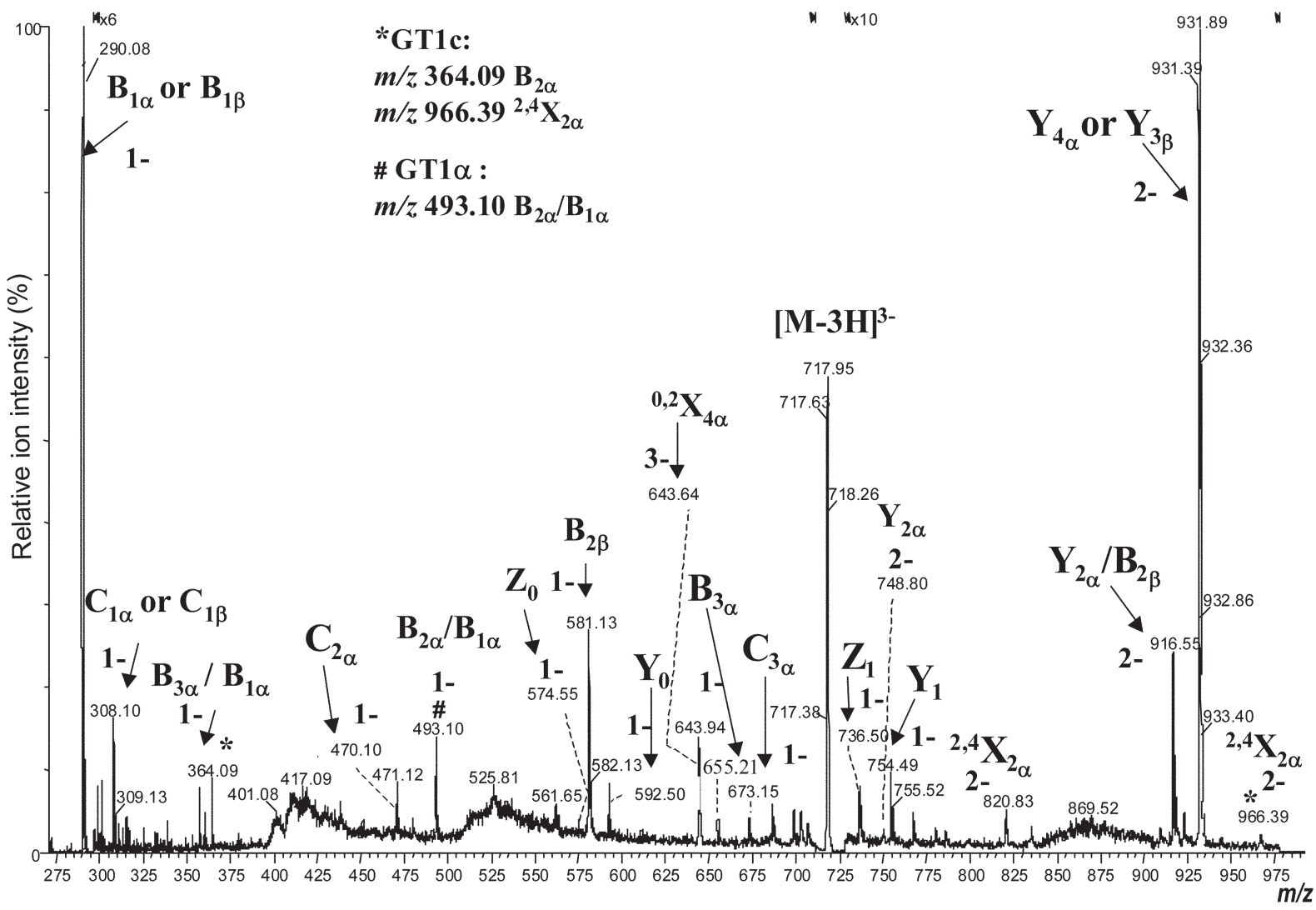

Fig. 5 Microchip ESI QTOF MS/MS of the triply charged ion at $m / z 717.50$ corresponding to GT1 (d18:1/20:0). ESI voltage $3 \mathrm{kV}$. Sampling cone potential $100 \mathrm{~V}$. The spectrum is derived by combining the scans corresponding to 40 and $70 \mathrm{eV}$ in the TIC MS/MS from Fig. 3b. The nomenclature for assignment of fragment ions is according to Domon and Costello nomenclature. ${ }^{31,32}$ 


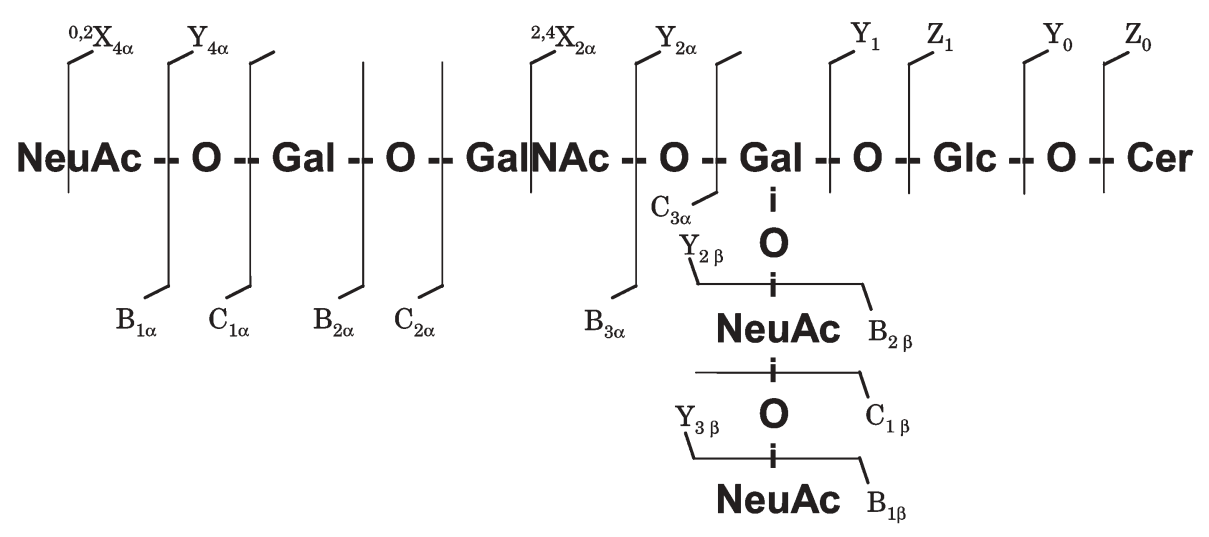

Fig. 6 The structure and the corresponding fragmentation scheme of the GT1b species. The product ions detected from the spectrum depicted in Fig. 5 are assigned.

even for differential biochemical diagnosis of certain clinically similar diseases i.e. differentiation of the three Schindler/ Kanzaki disease subgroups, clinically classified as type I, II and III.

The high sequencing efficiency of the microchip ESI QTOF MS/MS resulted in an information-rich fragmentation pattern. This feature is of particular importance for elucidating the presence of structural isomers or isobars as distinct species. In many cases these species play a particular physiological and/or pathological role and may, therefore, be of specific diagnostic relevance.

Alina D. Zamfir, $\dot{\dagger}^{* a}$ Niels Lion, $\grave{\dagger}^{b}$ Željka Vukelić, ${ }^{c}$ Laura Bindila, ${ }^{a}$ Joël Rossier, ${ }^{d}$ Hubert H. Girault ${ }^{b}$ and Jasna Peter-Katalinić $*^{* a}$ ${ }^{a}$ Institute for Medical Physics and Biophysics, Biomedical Analysis, University of Münster, Germany.E-mail: zamfir@uni-muenster.de; jkp@uni-muenster.de; Fax: +49-251-8355140; +49-251-8355194; Tel: +49-251-8352308

${ }^{b}$ Laboratoire d'Electrochimie Physique et Analytique, Ecole Polytechnique Federale de Lausanne, Switzerland

${ }^{c}$ Department of Chemistry and Biochemistry, Faculty of Medicine, University of Zagreb, Croatia

${ }^{d}$ DiagnoSwiss SA, Monthey, Switzerland

\section{References}

1 X. Czeszak, W. Morelle, G. Ricart, D. Tetaert and J. Lemoine, Anal. Chem., 2004, 76, 4324.

2 M. Wuhrer, C. H. Hokke and A. M. Deelder, Rapid Commun. Mass Spectrom., 2004, 18, 1741.

3 Y. O. Tsybin, M. Ramstrom, M. Witt, G. Baykut and P. Hakansson, J. Mass Spectrom., 2004, 39, 719.

4 J. E. Syka, J. J. Coon, M. J. Schroeder, J. Shabanowitz and D. F. Hunt, Proc. Natl. Acad. Sci. USA, 2004, 101, 9528.

5 N. G. Karlsson, B. L. Schulz and N. H. Packer, J. Am. Soc. Mass Spectrom., 2004, 15, 659.

6 S. Zhang, C. K. Van Pelt and J. D. Henion, Electrophoresis, 2003, 24, 3620 .

7 S. Zhang, C. K. Van Pelt and D. B. Wilson, Anal. Chem., 2003, 75, 3010.

8 C. K. Van Pelt, S. Zhang, E. Fung, I. Chu, T. Liu, C. Li, W. A. Korfmacher and J. Henion, Rapid Commun. Mass Spectrom., 2003, 17, 1573.

9 J. T. Kapron, E. Pace, C. K. Van Pelt and J. Henion, Rapid Commun. Mass Spectrom., 2003, 17, 2019.

10 J. M. Dethy, B. L. Ackermann, C. Delatour, J. D. Henion and G. A. Schultz, Anal. Chem., 2003, 75, 805.
11 A. Zamfir, S. Vakhrushev, A. Sterling, H. J. Niebel, M. Allen and J. Peter-Katalinić, Anal. Chem., 2004, 76, 2046.

12 S. Zhang and D. Chelius, J. Biomol Technol., 2004, 15, 120.

13 M. Schilling, W. Nigge, A. Rudzinski, A. Neyer and R. Hergenroder, Lab Chip, 2004, 4, 220.

14 K. Huikko, P. Ostman, K. Grigoras, S. Tuomikoski, V. M. Tiainen, A. Soininen, K. Puolanne, A. Manz, S. Franssila, R. Kostiainen and T. Kotiaho, Lab Chip, 2003, 3, 67.

15 M. Svedberg, M. Veszelei, J. Axelsson, M. Vangbo and F. Nikolajeff, Lab. Chip, 2004, 4, 322.

16 S. Le Gac, J. Carlier, J. C. Camart, C. Cren-Olive and C. Rolando, J. Chromatogr., B: Anal. Technol. Biomed. Life Sci., 2004, 808, 3.

17 V. Gobry, J. van Oostrum, M. Martinelli, T. C. Rohner, F. Reymond, J. S. Rossier and H. H. Girault, Proteomics, 2002, 2, 405 .

18 J. Rossier, N. Youhnovski, N. Lion, E. Damoc, S. Becker, F. Reymond, H. H. Girault and M. Przybylski, Angew. Chem., Int. Ed., 2003, 42, 53 .

19 Y. Yang, J. Kameoka, T. Wachs, J. D. Henion and H. G. Craighead, Anal. Chem., 2004, 76, 2568.

20 H. U. Linden, R. A. Klein, H. Egge, J. Peter-Katalinić, J. Dabrowski and D. Schindler, Biol. Chem. Hoppe Seyler, 1989, 370, 661.

21 O. P. van Diggelen, D. Schindler, W. J. Kleijer, J. G. M. Huijmans, H. Galjaard, H. U. Linden, J. Peter-Katalinić, H. Egge, U. Dabrowski and M. Cantz, Lancet, 1987, $2,804$.

22 W. Metelmann, Ž. Vukelić and J. Peter-Katalinić, J. Mass Spectrom., 2001, 36, 21.

23 J. Peter-Katalinić, Mass Spectrom. Rev., 1994, 13, 77.

24 J. Zaia, Mass Spectrom. Rev., 2004, 23, 161.

25 A. Zamfir and J. Peter-Katalinić, Electrophoresis, 2004, 25, 1949.

26 C. Thanawiroon, K. G. Rice, T. Toida and R. J. Linhardt, J. Biol Chem., 2004, 279, 2608.

27 J. S. Rossier, C. Vollet, A. Carnal, G. Lagger, V. Gobry, H. H. Girault, P. Michel and F. Reymond, Lab. Chip, 2002, 2, 145

28 V. Gobry, J. van Oostrum, M. Martinelli, T. Rohner, J. S. Rossier and H. H. Girault, Proteomics, 2002, 2, 405.

29 I. Meisen, J. Peter-Katalinić and J. Muthing, Anal. Chem., 2003, 75, 5719.

30 L. Bindila, R. Almeida, A. Sterling, M. Allen, J. Peter-Katalinić and A. Zamfir, J. Mass. Spectrom., 2004, 39, 1190.

31 B. Domon and C. E. Costello, Glycoconjugate J., 1988, 5, 397.

32 C. E. Costello, P. Juhasz and H. Perreault, Progr. Brain Res., 1994, 101, 45.

33 Ž. Vukelić, W. Metelmann, J. Müthing, M. Kos and J. PeterKatalinić, Biol. Chem., 2001, 382, 259.

34 A. Zamfir, Ž. Vukelić and J. Peter-Katalinić, Electrophoresis, 2002, 23, 2894.

35 A. Zamfir, Ž. Vukelić, L. Bindila, R. Almeida, A. Sterling, M. Allen and J. Peter-Katalinić, J. Am. Soc. Mass Spectrom., 2004, 15, 1649.

36 A. Zamfir, D. G. Seidler, H. Kresse and J. Peter-Katalinić, Glycobiology, 2003, 13, 733. 